\title{
An optical grating filter dry-etched on a $\mathrm{LiNbO}_{3}$ substrate
}

\author{
Satoshi Shinada ${ }^{\text {a)}}$, Tetsuya Kawanishi, Takahide Sakamoto, \\ and Masayuki Izutsu \\ National Institute of Information and Communications Technology \\ 4-2-1 Nukui-Kitamachi, Koganei-shi, Tokyo 184-8795, Japan \\ a)sshinada@nict.go.jp
}

Abstract: We fabricated an etched grating filter on a Ti-diffused waveguide in $\mathrm{LiNbO}_{3}$ using inductively coupled plasma etching with $\mathrm{C}_{4} \mathrm{~F}_{8} / \mathrm{Ar}$ as an etching gas, which has an etching rate of $85.1 \mathrm{~nm} / \mathrm{min}$. The etched grating filter had a reflectivity of $35 \%$ and a bandwidth of $0.02 \mathrm{~nm}$. Maximum reflectivity was obtained when the electric field of an incident beam was perpendicular to the grating.

Keywords: $\mathrm{LiNbO}_{3}, \mathrm{ICP}$, dry etching, grating

Classification: New functional devices and materials

\section{References}

[1] K. Noguchi, O. Mitomi, H. Miyazawa, and S. Seki, "A Broadband Ti:LiNbO 3 Optical Modulator with a Ridge Structure," J. Lightwave Technol., vol. 13, no. 6, pp. 1164-1168, June 1995.

[2] A. Chowdhury, C. Staus, B. F. Boland, T. F. Kuech, and L. McCaughan, "Experimental demonstration of 1535-1555-nm simultaneous optical wavelength interchange with a nonlinear photonic crystal," Opt. Lett., vol. 26, no. 17, pp. 1353-1355, Sept. 2001.

[3] Ch. Becker, A. Greiner, Th. Oesselke, A. Pape, W. Sohler, and H. Suche, "Integrated optical Ti:Er: $\mathrm{LiNbO}_{3}$ distributed Bragg reflector laser with a fixed photorefractive grating," Opt. Lett., vol. 23, no. 15, pp. 1194-1196, Aug. 1998.

[4] T. Kawanishi, K. Yoshiara, S. Oikawa, S. Shinada, T. Sakamoto, and M. Izutsu, "Integrated Reciprocating Optical Modulator for Optical HighOrder Sideband Generation," Jpn. J. Appl. Phys., vol. 43, no. 8B, pp. 5791-5794, Aug. 2004.

[5] S. Matsui, T. Yamato, H. Aritome, and S. Namba, "Microfabrication of $\mathrm{LiNbO}_{3}$ by Reactive Ion-Beam Etching," Jpn. J. Appl. Phys., vol. 19, no. 8, pp. L463-L465, Aug. 1980.

[6] K. Shima, N. Mitsugi, and H. Nagata, "Surface precipitates on single crystal $\mathrm{LiNbO}_{3}$ after dry-etching by CHF plasma," J. Mater. Res., vol. 13, no. 3, pp. 527-529, March 1998.

[7] K. Fukuda, T. Kai, and T. Sugita, "Fabrication of ridge-type waveguide in $\mathrm{MgO}: \mathrm{LiNbO}_{3}$ by using ICP Dry Etching," The 50th Spring Meeting 2003, JSAP, 29a-D-8, Yokohama, March 2003.

[8] T. Sugita, K. Mizuuchi, K. Yamamoto, K. Fukuda, T. Kai, I. Nakayama, and K. Takahashi, "Highly efficient second-harmonic generation in direct- 
bonded $\mathrm{MgO}: \mathrm{LiNbO}_{3}$ pure crystal waveguide," Electron. Lett., vol. 40, no. 21 , pp. 1359-1361, Oct. 2004.

\section{Introduction}

$\mathrm{LiNbO}_{3}(\mathrm{LN})$ is an attractive material with a high electro-optic coefficient and a low optical propagation loss. It has been used to develop various applications, such as a high-speed optical modulator, an optical sensor, a microwave filter, and a millimeter-wave generator. Additionally, new and improved characteristics of LN-based devices can be created by applying an etched structure to an LN substrate.

An etched ridge waveguide in an optical modulator controls the velocity mismatch between the optical wave and the modulating signal and concentrates the electric field on the waveguide, resulting in a large modulation bandwidth and a low driving voltage [1]. Also, the photonic crystal structure etched on the LN substrate produces the novel characteristics of a wavelength conversion or a spatial switch [2]. Etching LN substrates with a high degree of selectivity and some minor roughness on the surface are essential for good performance of the LN-based devices.

An optical filter with a periodical refractive index can be formed on an LN surface using a holographic technique with a laser that is a photorefractive grating [3]. However, its induced index change is unstable and can be restored by the passage of a high-power laser. We have developed an optical filter with an etched grating structure using electron-beam (EB) lithography and dry etching. The etched filters can be designed during EB lithography, and different band filters can be easily placed on a same surface. Our reciprocating optical modulator [4], which consists of an LN phase modulator and two fiber Bragg gratings (FBGs) as filters, can be constructed monolithically on an LN chip by replacing the FBGs with etched filters. It is important to prepare the precise periodical pattern on the LN surface and to use dry etching with a high degree of etching selectivity for etching the LN surface deeply. In this paper, we describe the characteristics of an optical grating filter that was fabricated using inductively coupled plasma (ICP) dry etching on an LN surface.

\section{Fabrication of the etched optical grating filter on LN}

Figure 1 shows the fabrication of an etched optical grating filter on LN. We prepared a z-cut and mirror finished 2.9-cm-long LN wafer with $10-\mu \mathrm{m}$ wide Ti-diffused optical waveguides. First, to prevent the LN surface from becoming charged during EB lithography, we coated a 10-nm-thick Ti film by surface sputtering. Then, an EB resist was formed on it, and a grating pattern was written on the resist by EB exposure. The period of the grating (between a line and a space) was $352 \mathrm{~nm}$, which corresponds to half the optical wavelength of $1550 \mathrm{~nm}$ in LN with a refractive index of 2.2. The patterns 


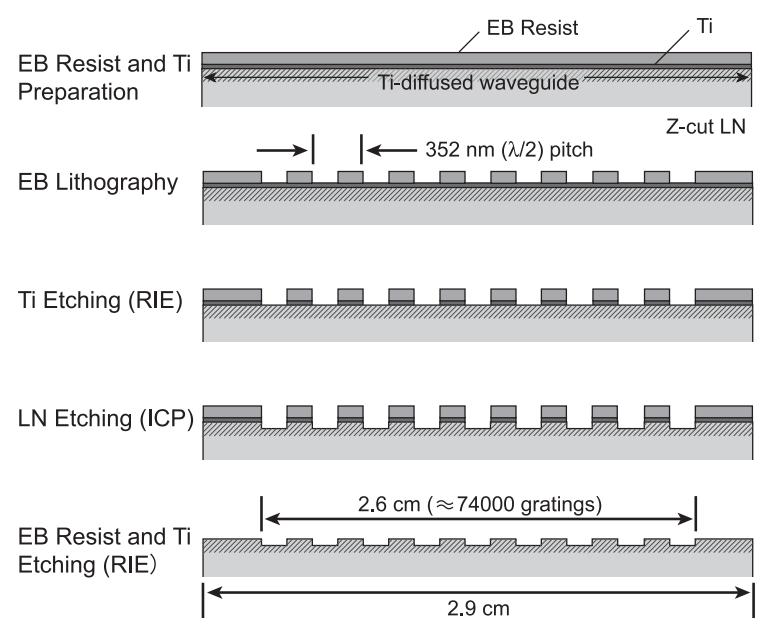

Fig. 1. Fabrication of etched grating filter on Ti-diffused waveguide in LN. Resist pattern of grating was formed using EB lithography, and LN was etched using ICP dry etching with $\mathrm{C}_{4} \mathrm{~F}_{8} / \mathrm{Ar}$ gas mixture.

were created during several tens of repetitions of lithography, and about three thousand gratings were written during each repetition. In total, about seventy-four thousand gratings spanned the length of 2.6-cm waveguides. Then, we etched the Ti film and LN substrate using reactive ion etching (RIE) and ICP etching, respectively. Finally, we removed the resist and Ti film using RIE. Fluorocarbons, such as $\mathrm{CF}_{4}$ and $\mathrm{CHF}_{3}$, are generally used as etching gasses for LN etching [5], but the etching rate of LN is lower than $\approx 100 \mathrm{~nm} / \mathrm{min}$, due to the deposition of lithium fluoride $(\mathrm{LiF})$ or carbon precipitates on the etched surface during the process [6]. We used a $\mathrm{C}_{4} \mathrm{~F}_{8} / \mathrm{Ar}$ gas mixture for the etching [7, 8]. The Ar gas was mixed into the etching gas in order to remove these precipitates. Furthermore, fluorocarbons with a high composition of carbon $\left(\mathrm{C}_{4} \mathrm{~F}_{8}\right)$ reduce the concentration of the fluorineradical present during etching, and as a result, the resist-etching rate can be kept low. An LN etching rate of $85.1 \mathrm{~nm} / \mathrm{min}$ and a selective etching rate of the LN to the resist mask of 1.57:1 were obtained.

\section{Characterization of the optical grating filter etched on LN}

Figure 2 shows an atomic force micrograph image of the ICP-etched grating on an LN surface. The distance between the bottom and top of the grating was $176 \mathrm{~nm}$, which was a good match for the design. The depth of the grating was about $8 \mathrm{~nm}$, and the roughness of the etched surface was minimal.

We measured the transmission characteristics of the Ti-diffused waveguide with the etched grating on it. Figure 3 (a) shows that the transparency of the emission depended on the wavelength of the incident light, and the inset shows the measurement setup. We used a tunable laser diode whose resolution is $0.001 \mathrm{~nm}$. A laser beam was coupled to the waveguide by a polarization-maintaining fiber, and the transmitted light was guided into the spectrum analyzer through a single-mode fiber. The polarization of the inci- 


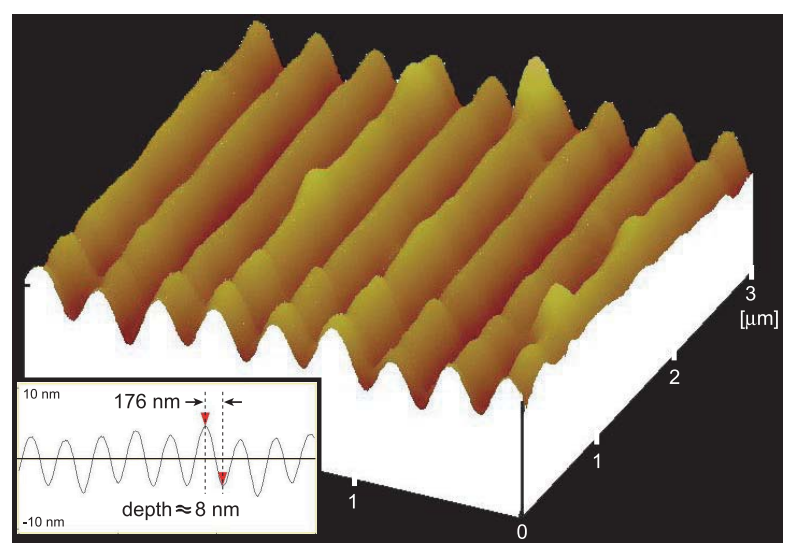

Fig. 2. Atomic force micrograph image of etched grating filter. Scanning area is $3 \times 3 \mu \mathrm{m}$.

(a)

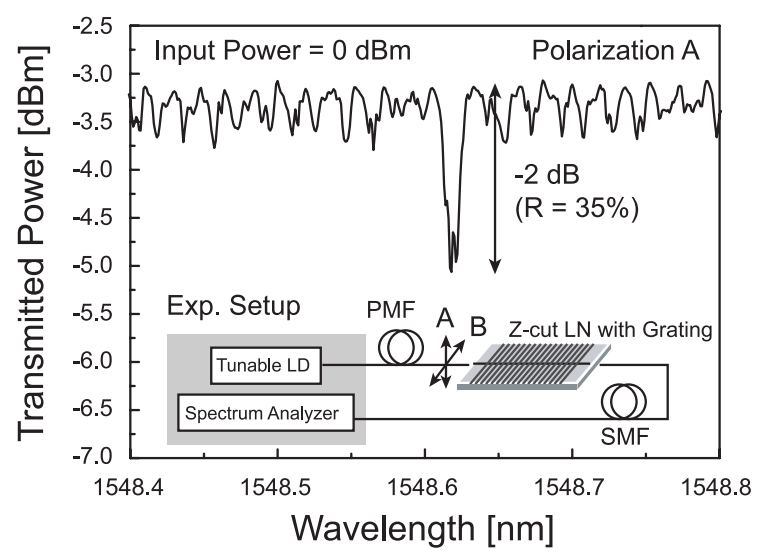

(b)

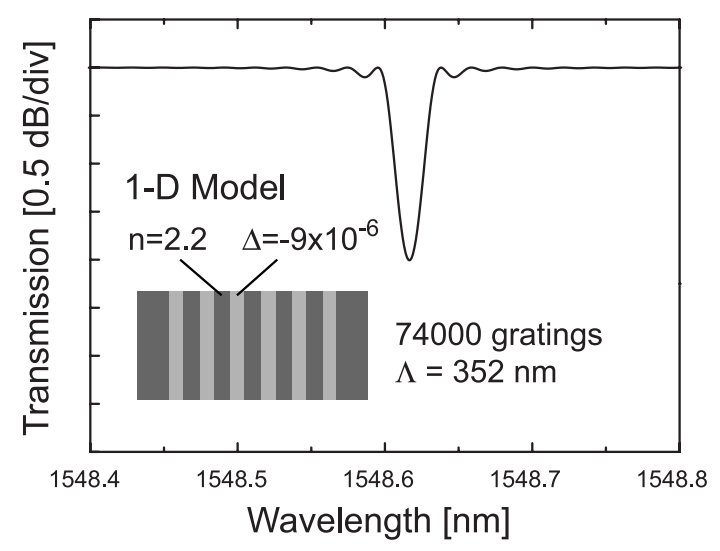

Fig. 3. (a) Transparency of waveguide with grating dependent on wavelength of incident light. Inset depicts measurement setup. (b) Calculated transmission for 1-D periodical model.

dent light was maintained in direction $A$, as shown in the inset of fig. 3(a). Its electric field was perpendicular to the grating (TE mode). A UV curing resin, with a refractive index of 1.6, was inserted between the fiber and the LN. The insertion loss was $3 \mathrm{~dB}$, and the ripple in Fig $3(\mathrm{a})$ was due to the reflection at the edge of the $\mathrm{LN}$ wafer. A decrease of $2 \mathrm{~dB}$ in transmitted power was obtained at the wavelength of $1548.62 \mathrm{~nm}$, and it corresponded to 
a reflectivity of $35 \%$. The reflection bandwidth was $0.02 \mathrm{~nm}$. From the fitting of the calculation using the one-dimensional periodical model, as shown in Fig. 3 (b), we determined that the equivalent refractive index difference between the LN layer $(n=2.2)$ and the etched layer was as small as $-10^{-5}$.

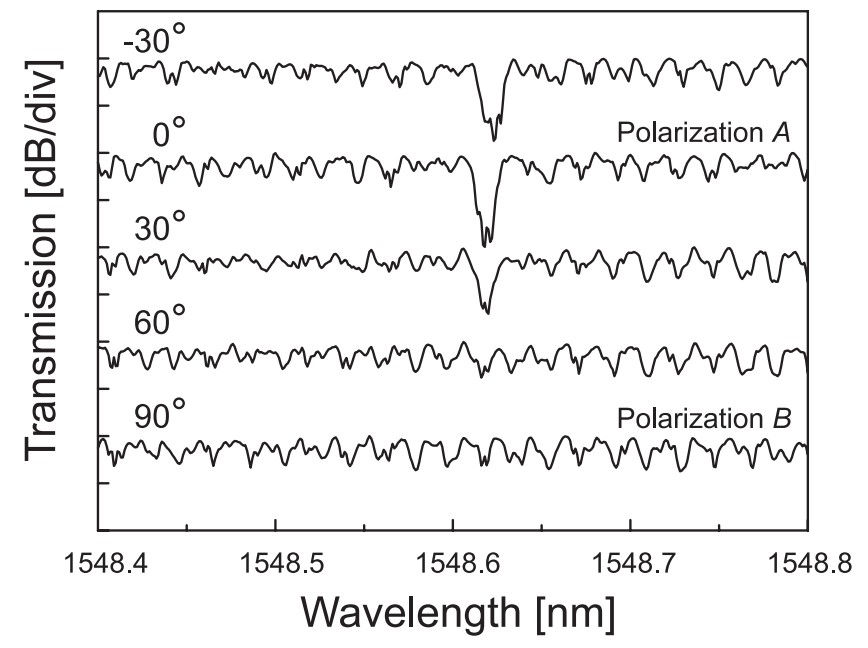

Fig. 4. Transparency depends on polarization of incident light. Zero and 90 degrees indicate directions of $\mathrm{A}$ and $\mathrm{B}$, respectively, in the inset of Fig. 3 (a).

Figure 4 shows that the transparency of the emission depended on the polarization of the incident light. The direction of polarization was rotated every 30 degrees. When the direction of the electric field was parallel to the grating (direction $B$ ), a reflection was not observed. A larger reflectivity and a wider reflection band can be obtained using a deeper grating. In future work, we will improve EB lithography for a thicker resist mask and ICP dry etching for higher selectivity.

\section{Conclusion}

We fabricated an etched optical grating filter on an LN surface with optical waveguides using ICP dry etching with $\mathrm{C}_{4} \mathrm{~F}_{8} / \mathrm{Ar}$ as the etching gas. Etching rate of $85.1 \mathrm{~nm} / \mathrm{min}$. and selective etching rate of the $\mathrm{LN}$ to the resist mask of 1.57:1 were obtained. The resist pattern of the seventy-four thousand gratings was formed by several tens of repetitions of EB lithography. The grating filter had a reflectivity of $35 \%$ and a reflection bandwidth of $0.02 \mathrm{~nm}$, and deeper etching would produce greater reflectivity. We determined that the reflectivity depends on the polarization of the incident beam. ICP dry etching with high etching rate and high selectivity is essential in various applications of LN.

\section{Acknowledgments}

The authors gratefully acknowledge T. Kai of Matsushita Electric Industrial Co., Ltd. for his co-operation in the ICP dry etching and M. Tsuchiya of 
NICT and K. Nishikawa of Kyosemi for their useful discussions. This work was supported by the Japan Society for the Promotion of Science (JSPS) with a grant-in-aid for scientific research (B) (\#16360183). 